\section{Hospital seguro frente aos desastres: uma reflexão sobre biossegurança e arquitetura}

\author{
Lucia Cristina de Paiva Saba, ${ }^{1}$ \\ Telma Abdalla de Oliveira Cardoso ${ }^{2}$ \\ e Marli B. M. de Albuquerque Navarro ${ }^{2}$
}

Como citar: Saba LCP, Cardoso TAO, Navarro MBMA. Hospital seguro frente aos desastres: uma reflexão sobre biossegurança e arquitetura. Rev Panam Salud Publica. 2012:31(2):176-80.

\section{SINOPSE}

Um dos maiores desafios da sociedade atual é o enfrentamento das adversidades causadas pelos desastres. Os estabelecimentos de saúde, principalmente os hospitais, são considerados essenciais nessas situações. Este trabalho discute os princípios da arquitetura do hospital seguro frente aos desastres, como propõem a Organização Mundial da Saúde e a Organização Pan-Americana da Saúde. Projetar um hospital seguro exige ações multidisciplinares, com envolvimento de administradores, arquitetos, engenheiros, médicos e enfermeiros. O planejamento de cada hospital pressupõe uma análise de riscos e aspectos de segurança específicos. Também é importante agregar a biossegurança ao conceito de hospital seguro. O equilibrio entre aspectos arquitetônicos e biossegurança permite a compreensão dos riscos associados ao trabalho, facilitando o dimensionamento de espaços para suportar as ações de resposta frente às emergências. Em suma, a programação de um hospital seguro requer uma sintese de conhecimentos que relacionam diversos saberes, entre eles os da biossegurança e da arquitetura hospitalar. Esses princípios devem embasar as indagações sobre o hospital seguro e o planejamento de projetos arquitetônicos com foco na manutenção das instalações em capacidade máxima mesmo diante de situações adversas.

Palavras-chave: risco sanitário; exposição a agentes biológicos; arquitetura hospitalar; planificación en desastres.

1 Fundação Oswaldo Cruz (Fiocruz), Instituto de Pesquisa Clínica Evandro Chagas, Rio de Janeiro (RJ), Brasil.

2 Fiocruz, Escola Nacional de Saúde Pública, Núcleo de Biossegurança, Rio de Janeiro (RJ), Brasil. Correspondência: Telma Abdalla de Oliveira Cardoso, abdalla@fiocruz.br
A Organização Pan-Americana da Saúde (OPAS) vem trabalhando intensamente em uma política internacional para reduzir os riscos em saúde advindos dos desastres. Como resultado, desenvolveu-se o programa de hospital seguro, que prevê hospitais com funcionamento garantido em situações de emergência e na fase de recuperação dessas emergências (1). O hospital seguro é essencial para o atendimento da população, considerando também a qualidade ambiental através do monitoramento de fatores de risco.

A industrialização propiciou transformações socioambientais que favorecem desigualdades econômicas e inchamento das cidades e têm impacto em territórios, padrões de consumo e geração de resíduos, entre outros. Esse cenário caracteriza contextos de risco cujas dimensões são verificadas nas análises do aquecimento global e na emergência e reemergência de doenças. Ainda, dado esse cenário, um dos maiores desafios atuais, segundo o Painel Intergovernamental sobre as Alterações Climáticas (Intergovernmental Panel on Climate Change, IPCC), estabelecido pelo Programa das Nações Unidas para o Meio Ambiente (PNUMA), é o enfrentamento das adversidades causadas pelos desastres, transformando o risco numa preocupação de todos e de cada um (2).

Com o objetivo de entender a relação entre desenvolvimento, risco e desastres, o Programa das Nações Unidas para o Desenvolvimento (United Nations Development Programme, UNDP) propôs um índice de risco de desastres (disaster risk index, DRI). O DRI mensura e compara os níveis relativos de exposição, vulnerabilidade e risco, identificando as vulnerabilidades específicas de cada país frente a três tipos de desastres: ciclones tropicais, secas e enchentes. Valoriza a construção de escalas nacionais para visualizar os padrões de risco, orientando o seu gerenciamento e mudanças na política de desenvolvimento e planejamento local (3).

De acordo com o DRI, a magnitude do risco é definida pela combinação entre exposição e vulnerabilidade, sendo a vulnerabilidade um conjunto de variáveis demográficas, socioeconômicas, técnicas ou ambientais que comprometem a capacidade da população de absorver o impacto de um evento perigoso e se recuperar dele (4). Um maior risco também está ligado a frequência, severidade, extensão ou imprevisibilidade dos desastres. $\mathrm{O}$ investimento em infraestrutura, no escopo do planejamento para o enfrentamento de desastres, é prioritário, pois permite respostas imediatas, viabilizadas pelas medidas de estabilidade das edificações, garantindo o controle operacional e do risco.

Os estabelecimentos de saúde, principalmente os hospitais, são considerados essenciais frente aos 
TABELA 1. Principais desastres ocorridos na América Latina e Caribe de 2000 a 2010

\begin{tabular}{|c|c|c|c|}
\hline Ano & Desastre & Local & Consequências \\
\hline \multirow[t]{2}{*}{2001} & Terremoto (6,9 na escala Richter) & Peru & 87 hospitais e serviços de saúde danificados ou destruídos \\
\hline & Terremoto (7,6 na escala Richter) & El Salvador & $\begin{array}{l}\text { Perda de } 39,1 \% \text { dos leitos hospitalares do país, cinco hospitais } \\
\text { danificados ou destruídos }\end{array}$ \\
\hline 2002 & Chuvas torrenciais e tempestade de granizo & Bolívia & Um hospital danificado \\
\hline 2003 & Inundação & Argentina & Dois hospitais e 63 serviços de saúde severamente danificados \\
\hline \multirow[t]{3}{*}{2004} & Furacão Ivan (categoria 3) & Granada & Dois hospitais, seis serviços de saúde danificados ou destruídos \\
\hline & Furacão Frances (categoria 3) & Bahamas & Cinco hospitais e serviços de saúde danificados ou destruídos \\
\hline & Furacão Jeanne (categoria 1) & $\begin{array}{l}\text { Bahamas } \\
\text { Haiti }\end{array}$ & $\begin{array}{l}\text { Cinco hospitais e serviços de saúde danificados ou destruídos } \\
\text { Um hospital inundado }\end{array}$ \\
\hline 2005 & Inundação & Guiana & 18 hospitais e serviços de saúde danificados ou destruídos \\
\hline 2006 & Inundação & Suriname & Quatro hospitais e serviços de saúde danificados ou destruídos \\
\hline 2007 & Terremoto ( 8,0 na escala Richter) & Peru (Pisco) & $\begin{array}{l}\text { 97\% dos leitos hospitalares perdidos, } 595 \text { mortos, } 1295 \text { pacientes } \\
\text { evacuados }\end{array}$ \\
\hline 2009 & Inundações e deslizamentos & Peru & $\begin{array}{l}30 \text { mortos, } 139000 \text { pessoas afetadas e um centro de saúde } \\
\text { atingido }\end{array}$ \\
\hline \multirow[t]{3}{*}{2010} & Furacão Ida (tempestade) & $\begin{array}{l}\text { El Salvador } \\
\text { Guatemala }\end{array}$ & $\begin{array}{l}192 \text { mortos, } 75000 \text { evacuadas e } 25 \text { serviços de saúde afetados } \\
158 \text { mortos, } 144355 \text { pessoas evacuadas, } 193113 \text { afetadas e } 22 \\
\text { serviços de saúde afetados }\end{array}$ \\
\hline & Terremoto (6,9 na escala Richter) & Chile & $\begin{array}{l}421 \text { mortos, } 61117 \text { desabrigados, } 758044 \text { afetados e } 26 \text { hospitais } \\
\text { danificados }\end{array}$ \\
\hline & Terremoto ( 7,0 na escala Richter) & Haiti & 220000 mortos, 300000 feridos e 30 hospitais danificados \\
\hline
\end{tabular}

Fonte: World Health Organization/Pan American Health Organization (6, 8), Organização Mundial da Saúde/Organização Pan-Americana da Saúde (7), World Health Organization/Collaborating Centre for Research on the Epidemiology of Disasters (9) e Brasil (10).

desastres (5). Em 2009, mais de 67\% dos quase 18000 hospitais da América Latina e do Caribe estavam localizados em áreas de risco elevado de desastre. Nos últimos 25 anos, muitos se tornaram inservíveis; mais de 45 milhões de pessoas pararam de receber assistência, e a perda econômica pela destruição de instalações e equipamentos ultrapassou 4 bilhões de dólares (6-10). A tabela 1 mostra os principais desastres ocorridos na América Latina e Caribe de 2000 a 2010.

O elenco de vulnerabilidades em uma situação de desastre e seu efeito na edificação hospitalar provocam reflexões sobre soluções projetuais de arquitetura e de engenharia como instrumentos essenciais ao desempenho das edificações. Quando um hospital é impactado por desastre, os efeitos de longo prazo ultrapassam as implicações da impossibilidade de tratar vítimas, uma vez que combinam alto valor econômico e alto valor social $(11,12)$. O comprometimento infraestrutural desses estabelecimentos está geralmente relacionado a projetos inadequados, falta de manutenção e localização em zonas de risco (13).

Em 2004, a OPAS e a Organização Mundial da Saúde (OMS) incentivaram os países a fortalecer sua capacidade de preparação e de mitigação de desastres através da adoção do lema "Hospital seguro frente aos desastres" como política de redução de riscos, com alocação de recursos para assegurar que o setor saúde permaneça operativo (em especial aquelas instalações que oferecem atenção primária), além de estabelecer que todas as novas unidades assistenciais sejam construídas com um nível de proteção, garantindo a continuidade funcional em situações de desastre (13).

Na 2a ${ }^{-}$Conferência Mundial sobre Redução de Desastres, realizada em 2005, no Japão, foi elaborado um plano de ação de orientação política para o período de 2005 a 2015 (11). O primeiro passo para implementar as ações foi definir o hospital seguro como uma "instalação de saúde cujos serviços permaneçam acessíveis e em operação com sua capacidade máxima, na mesma infraestrutura, durante e imediatamente após o impacto de um perigo natural" (p. 1) (14). Nessa Conferência, foi lançada a Campanha Mundial de Redução de Desastres para 2008/2009, com a Estratégia Internacional das Nações Unidas para a Redução de Desastres (EIRD), onde se inseria a campanha de hospitais seguros, mostrando a importância dessas edificações e de sua atuação em emergências, estabelecendo-se então o programa "Hospitais seguros frente aos desastres".

Este trabalho discute os princípios da arquitetura do hospital seguro e tece considerações acerca da biossegurança nesse contexto.

\section{PLANEJAMENTO, VULNERABILIDADES E BIOSSEGURANÇA NO HOSPITAL SEGURO}

As diretrizes do planejamento para um hospital seguro recomendam ações multidisciplinares, com ênfase na atuação de diversos atores, como administradores, arquitetos, engenheiros, médicos e enfermeiros, entre outros (15). A OMS formulou três critérios fundamentais para orientar esse planejamento: proteção da vida, investimentos e função, ou seja, a edificação deve ser capaz de manter-se de pé, resistir com danos mínimos aos impactos dos fenômenos destrutivos e continuar operando, mantendo sua produção de serviços de saúde como parte da rede à qual pertence (16).

As vulnerabilidades relacionadas aos aspectos de engenharia da edificação hospitalar dividem-se em vulnerabilidades estruturais e não estruturais. As estruturais se referem às partes que sustentam a edifica- 
ção, como lajes, vigas, muros, composição do concreto e armadura de ferro. As não estruturais se referem a componentes associados aos elementos estruturais, como paredes, teto, janelas, portas, esquadrias, painéis de vidro e telhados, e a componentes que cumprem funções essenciais, como instalações elétricas, hidráulicas, fluido-mecânicas, sanitárias, gases e ar condicionado, ou ainda que compõem o interior da edificação, como móveis e equipamentos mecânicos e médicos. Destacam-se, entre os itens não estruturais: os sistemas de ventilação, que permitem a renovação do ar (controle de transmissão de doenças); o abastecimento contínuo de água (existência de cisterna ou tanque em planos elevados com autonomia de 48 horas); o abastecimento de energia elétrica permanente (necessidade de geradores), uma vez que, durante desastres, são frequentes os cortes de energia; luzes de emergência (autonomia mínima de 4 horas, para facilitar o escape); e existência de portas contra fogo (abertura no sentido do fluxo de saída).

Há ainda a vulnerabilidade administrativoorganizativa (fatores funcionais), ligada a segurança da edificação, distribuição e relação dos espaços arquitetônicos, dos serviços médicos e de apoio (disponibilidade de água, eletricidade, insumos, etc.), a processos administrativos (contratos, compras, manutenção predial e de equipamentos, entre outros) e a relações de dependência física e funcional entre as diferentes áreas de um hospital (rotas de acesso e fluxos). A adequada separação das áreas por zonas e a relação entre tais zonas garantem o funcionamento do hospital em casos de emergência, assim como a preparação do estabelecimento de saúde através de planos e procedimentos de emergência até exercícios de simulação $(7,15)$. Deve-se ressaltar que o colapso de hospitais com danos materiais e humanos não é determinado somente pelo fenômeno natural, mas está, em geral, vinculado à ineficiência ou deficiência do fluxo operacional, à desconsideração dos perigos naturais para a edificação e à falta de manutenção dos sistemas e equipamentos $(7,8)$.

É interessante notar que as diretrizes para a programação do hospital seguro mostram um distanciamento dos conceitos de biossegurança. Na implantação do hospital seguro, a biossegurança relaciona-se tão somente à epidemiologia dos desastres, visando ao controle das doenças decorrentes. Entretanto, o espaço físico combina elementos construtivos e métodos preventivos de manutenção da qualidade ambiental, contribuindo para reduzir ou eliminar a possibilidade de ocorrência de efeitos adversos pela liberação acidental ou intencional de agentes causais de desequilíbrios ambientais ou de risco que possam causar impactos à saúde pública ou ao ambiente (17). Na perspectiva do hospital seguro, a biossegurança pode contribuir diretamente para o cumprimento dos critérios fundamentais propostos pela OMS para o hospital seguro.

A biossegurança, campo relativamente novo, muitas vezes não é valorizada pela arquitetura, pela dificuldade de troca entre saberes, favorecida pela cultura cartesiana. Entretanto, novas diretrizes de monitoramento de risco exigem um esforço conjunto dos profissionais envolvidos no planejamento do projeto arquitetônico e o estabelecimento de padrões que assegurem as condições de segurança necessárias, modificando as concepções de espaços, materiais de acabamento, mobiliário, tratamento e renovação do ar para minimizar os riscos inerentes às atividades de assistência e de pesquisa (18).

Os níveis de biossegurança são combinações de critérios de contenção. Cada combinação é determinada pela avaliação de risco e proporciona níveis crescentes no grau de contenção e na complexidade do nível de proteção (18). Considerando-se que os setores dos hospitais convivem constantemente com diversos agentes de risco, destacando-se, por sua importância e premência, o risco biológico, a aplicação de ações preventivas centradas na discussão dos princípios de contenção torna-se essencial na abordagem sobre as condições de trabalho em um ambiente hospitalar sustentável e seguro. Podemos exemplificar essa essencialidade com a implantação de barreiras de contenção relacionadas ao sistema de ar condicionado em áreas assistenciais de pacientes com doenças de transmissão aérea.

O marco legal da biossegurança no Brasil foi a Lei 8 974/1995, voltada para a tecnologia de engenharia genética (19). Em 2002, o Ministério da Saúde constituiu a Comissão de Biossegurança em Saúde (Portaria 343), ampliando o foco de atuação legal vigente e o fortalecimento de políticas institucionais de biossegurança, a fim de orientar ações que alcançassem o domínio da arquitetura nos serviços de saúde. A Comissão definiu biossegurança como "condição de segurança alcançada por um conjunto de ações destinadas a prevenir, controlar, reduzir ou eliminar riscos inerentes às atividades que possam comprometer a saúde humana, animal e vegetal e o meio ambiente" (20).

$\mathrm{O}$ equilíbrio entre as exigências projetuais e a biossegurança dirigirá a compreensão dos riscos associados ao trabalho, permitindo, dessa forma, um novo olhar que alcance o hospital seguro, integrando os procedimentos e a atenção médica aos espaços dimensionados para suportar as ações de respostas frente às emergências.

\section{CONTEXTO E MITIGAÇÃO}

Os dados do banco EM-DAT, do Centro de Pesquisa sobre a Epidemiologia dos Desastres (Centre for Research on the Epidemiology of Disasters, CRED), apontam o Brasil como um dos países mais afetados por inundações e enchentes. Entre 1960 e 2008 registraramse no Brasil 94 desastres, 5720 mortes e 15 milhões de desabrigados e desalojados. O país, em 2008, ocupou o $10^{\circ}$ lugar no ranking mundial de desastres hidrológicos (enchentes, inundações, deslizamentos), com 1,8 milhão de vítimas $(9,21)$.

A relação entre ambiente deteriorado, processo de urbanização caótico, vulnerabilidade e desastres naturais tornou-se mais expressiva nos últimos 50 
anos, principalmente nos países em desenvolvimento. A ocupação de áreas de risco, como encostas e proximidade de rios, o acúmulo de lixo nos corpos hídricos, os fluxos migratórios intensos, a precariedade das construções sem avaliação técnica quanto aos cortes e aterro dos terrenos, a devastação de ecossistemas e a exploração predatória dos recursos naturais, o aumento da pobreza, a ausência de políticas públicas e a ineficiência dos serviços de infraestrutura urbana evidenciam a fragilidade das populações frente aos desastres (22). Essas vulnerabilidades se manifestam em dois sentidos: primeiro, em uma dimensão cada vez maior das áreas suscetíveis a danos causados pelos desastres; segundo, na generalização e no acúmulo de vulnerabilidades a partir das condições de crescimento de determinados setores sociais urbanos (23).

As considerações sobre os critérios para projetos de arquitetura demonstram que cada edificação hospitalar, em função de suas complexidades, assume um aspecto próprio; dessa forma, para cada hospital planejado, uma composição de análises e pesquisas envolvendo o risco das atividades e conceitos de segurança contribui para a formalização de um projeto hospitalar seguro. A importância desse aspecto se destaca diante das inundações e deslizamentos ocorridos em janeiro de 2011 na região serrana do Estado do Rio de Janeiro, atingindo 18 municípios. Os levantamentos iniciais informaram que em torno de 95000 pessoas haviam sido atingidas, com 10484 desabrigados, 24198 desalojados e 916 óbitos. Os serviços de saúde desses municípios foram severamente castigados (10).

Portanto, os projetos para reduzir riscos exigem etapas orientadoras. A primeira relaciona-se às ações preventivas, para eliminar riscos, evitando a ocorrência do desastre. A segunda, direcionada à diminuição da magnitude do evento, reduz danos, como perdas humanas e materiais $(24,25)$, e compreende medidas de engenharia, econômicas, administrativas, políticas e sociais $(26,27)$. Essas medidas devem incluir critérios para o planejamento e a construção de edificações hospitalares onde a exposição a risco seja inexpressiva. Tais edificações devem ser distanciadas de zonas costeiras de alto risco ou sujeitas a deslizamentos e inundações. Também não devem ocupar terrenos íngremes, instáveis e de terra solta; e devem ficar afastados de grandes centros industriais e de outras situações ameaçadoras. Além disso, deve haver reforço estrutural das edificações existentes que abrigam hospitais, escolas, sistemas de água potável e energia. Os critérios de mitigação devem ser aplicados aos edifícios existentes, otimizando a qualidade e a funcionalidade dessas construções, para que possam suportar qualquer tipo de ameaça.

Um desenvolvimento econômico mais equitativo é essencial para a mitigação de desastres. Os planos de mitigação devem integrar as políticas governamentais, em todos os níveis, independentemente das alterações políticas, orçamentárias e administrativas. Precisam possuir prioridade local e base institucional para implementação, incluindo a criação de plataformas multissetoriais para orientar os processos de for- mulação de políticas e coordenar as ações de resposta; integração com as políticas de desenvolvimento; e participação da comunidade, a fim de que as necessidades locais sejam atendidas. É necessária, concomitantemente, a criação de fundos de emergência para prestar apoio às atividades de preparação, resposta e recuperação frente aos desastres.

As medidas de mitigação de desastres só serão efetivas se a sociedade estiver consciente da dimensão dos eventos aos quais está exposta. É importante a socialização de informações dentro de estratégias de educação.

\section{CONSIDERAÇÕES FINAIS}

A programação de um hospital seguro é evidenciada por uma síntese de conhecimentos que relacionam diversos saberes, entre eles os da biossegurança e da arquitetura hospitalar. O planejamento hospitalar baseado em questões multidisciplinares busca a centralidade e o desenvolvimento de soluções arquitetônicas com foco na promoção da inter-relação como ferramenta fundamental para as atividades hospitalares. Os conceitos de biossegurança e arquitetura hospitalar devem reforçar as indagações e propostas projetuais sobre o hospital seguro, ressaltando a relevância de suas aplicabilidades relacionadas com as políticas de implantação.

Os atores envolvidos na proposição do hospital seguro devem priorizar a eficiência e a sustentabilidade do projeto da edificação e de suas operações frente a um desastre. A concepção de um projeto arquitetônico deve resultar da análise dos processos que ocorrem no ambiente, gerando um planejamento que perceba quais interferências podem comprometer as ações, relacionando espaço construído, usuários, funcionários e o entorno, integrando competências multidisciplinares. A estratégica relação entre a biossegurança e o planejamento arquitetônico amplia a qualidade projetual, diminuindo fragmentações na concepção do edifício hospitalar em suas complexidades.

\section{SYNOPSIS}

\section{Hospitals safe from disasters: a reflection on architecture and biosafety}

One of the biggest challenges in today's society is facing adversity caused by disasters. Health facilities, especially hospitals, are considered essential in these situations. This article discusses the principles of architectural design of hospitals safe from disasters, as proposed by the World Health Organization and the Pan American Health Organization. Designing a safe hospital requires multidisciplinary efforts, involving administrators, architects, engineers, physicians, and nurses. The planning of each hospital demands the analysis of specific risks and safety concerns. The concept of biosafety should also be addressed in plan- 
ning safe hospitals. The balance between architectural aspects and biosafety provides an understanding of workassociated risks, facilitating the adequate planning of spaces to support response actions to emergencies. In short, the planning of a safe hospital requires the synthesis of various types of expertise, including those relating to biosafety and architecture. These principles should support the appraisal of safe hospitals and architectural planning with a focus on preparing facilities to function at full capacity even in the face of adverse situations.

Key words: health risk; exposure to biological agents; hospital design and construction; disaster planning.

\section{REFERÊNCIAS}

1. SEGOB - Sistema Nacional de Protección Civil. Guía Práctica del Programa Hospital Seguro. Disponível em: http:/ / www.proteccioncivil.gob.mx/work/ models/ProteccionCivil/Resource/59/ 1/images/gpphs.pdf Acessado em 14 de dezembro de 2010.

2. United Nations, Intergovernmental Panel on Climate Change. Climate change 2007: synthesis report. An assessment of the Intergovernmental Panel on Climate Change. Disponível em: http://www.ipcc.ch/pdf/assessment-report/ar4/syr/ar4_syr.pdf Acessado em 20 de agosto de 2011.

3. United Nations Development Programme. Reducing disaster risk: a challenge for development. Nova Iorque: UNDP; 2004. Disponível em: http:// www.undp.org/cpr/whats_new/rdr english.pdf Acessado em 20 de agosto de 2011.

4. Braga TM, Oliveira EL, Givisiez GHN. Avaliação de metodologias de mensuração de risco e vulnerabilidade social a desastres naturais associados à mudança climática. Sao Paulo Perspect. 2006;20(1):81-95.

5. Federal Emergency Management Agency. Applicant handbook. Washington: FEMA; 1999.

6. World Health Organization, Pan American Health Organization. Guidelines for vulnerability reduction in the design of new health facilities. Washington: PAHO; 2004

7. Organización Mundial de la Salud, Organización Panamericana de la Salud, $49^{\circ}$ Consejo Directivo, 61로 Sesión del Comité Regional. Informe de la mesa redonda sobre hospitales seguros: una meta a nuestro alcance. CD49/22, 01/10/2009. Disponível em: http://new.paho.org/ per $/$ index.php?gid $=168 \&$ option $=$ com docman\&task=doc_download Acessado em 20 de agosto de 2011.

8. World Health Organization, Pan American Health Organization. 2009 annual report: emergency preparedness and disaster relief. Washington: OMS; 2009.

9. World Health Organization, Collaborating Centre for Research on the Epidemiology of Disasters (CRED). The International Disaster Database. Disponível em: http://www.emdat.be Acessado em 3 de janeiro de 2011.
10. Brasil, Secretaria Nacional de Defesa Civil [Internet]. Disponível em: http:// www.defesacivil.gov.br Acessado em 21 de janeiro de 2011.

11. Organização das Nações Unidas, Estratégia Internacional para a Redução de Desastres. Marco de Ação de Hyogo para 2005-2015: aumento da resiliência das nações e das comunidades frente aos desastres. Em: Conferência Mundial sobre a Redução de Desastres; 18 a 22 de janeiro de 2005; Kobe, Japão. Genebra: ONU; 2005.

12. Organización Mundial de la Salud, Organización Panamericana de la Salud. Hospitales seguros ante inundaciones. Washington: OPS; 2006.

13. Organização Mundial da Saúde, Organização Pan-Americana da Saúde, $45^{\circ}$ Conselho Diretor, 56 $6^{\text {a }}$ Sessão do Comitê Regional. Resolução CD45.R8: Capacidade de preparação e resposta a desastres. Washington: OPS; 2004. Disponível em: http://www.paho.org/ portuguese/gov/cd/cd45.r8-p.pdf Acessado em 20 de agosto de 2011.

14. Organização Mundial da Saúde, Organização Pan-Americana da Saúde, 140 a Sessão do Comitê Executivo. CE140/13: Iniciativa regional de hospitais seguros. Washington: OPAS; 2007. Disponível em: http://www.paho.org/portu guese/gov/ce/ce140-13-p.pdf Acessado em 20 de agosto de 2011.

15. Organización Mundial de la Salud, Organización Panamericana de la Salud. Fundamentos para la mitigación de desastres en establecimientos de salud. Washington: OPS; 2000.

16. Organización Mundial de la Salud, Organización Panamericana de la Salud. Hospitales seguros: recomendaciones prácticas. Washington: OPS; 2004.

17. Simas CM, Cardoso TAO. Arquitetura e biossegurança. Em: Teixeira P, Valle S, eds. Biossegurança: uma visão multidisciplinar. Rio de Janeiro: Fiocruz; 2011. Pp. 75-110.

18. Simas CM, Cardoso TAO. Biossegurança e arquitetura em laboratórios de saúde pública. Pos Rev Programa Pos-Grad Arquit Urban FAUUSP. 2008;24:108-24.

19. Brasil. Lei $8974 / 1995$. Diário Oficial da União, Brasília, v. 133, n. 5, p. 337-346, SeçãoI. Disponívelem: http://legislacao. planalto.gov.br/legisla/legislacao.nsf/
Viw_Identificacao/lei\%208.974-1995? OpenDocument Acessado em 2 de outubro de 2011.

20. Brasil, Ministério da Saúde, Comissão de Biossegurança em Saúde. Diretrizes para o trabalho em contenção com material biológico. Brasília: Ministério da Saúde; 2004.

21. Tominaga LK, Santoro J, Amaral R, orgs. Desastres naturais: conhecer para prevenir. São Paulo: Instituto Geológico; 2009.

22. Martinez EA, La Rosa FL, Escobar PM. Vulnerabilidad en establecimientos de salud. Lima: Organización Mundial de la Salud, Organización Panamericana de la Salud; 1996.

23. Mansilla E. Riesgo y ciudad. Cidade do México: Universidad Nacional Autónoma de México; 2000.

24. Organización Mundial de la Salud, Organización Panamericana de la Salud. Hacia un mundo más seguro frente a los desastres naturales: la trayectoria de América Latina y El Caribe. Washington: OPS; 1994

25. Organización Mundial de la Salud, Organización Panamericana de la Salud. Manual de evaluación de daños y necesidades en salud para situaciones de desastre. Ecuador: OPS; 2004. (Serie Manuales y Guías sobre Desastres, № 4 .)

26. Lourdes A, Justiniani H, Gaspar O, Henríquez E. Vulnerabilidad de las instituciones de salud ante la ocurrencia de desastres naturales en la República de Panamá. Panamá: Universidad Tecnológica de Panamá; 1998.

27. Organización Mundial de la Salud, Organización Panamericana de la Salud, Oficina Regional de la Organización Mundial de la Salud. Hospitales seguros: una responsabilidad colectiva, un indicador mundial de reducción de los desastres. Washington: OPS; 2005.

Manuscrito recebido em 28 de janeiro de 2011. Aceito em versão revisada em 2 de junho de 2011. 